\title{
Virginia Zavala*
}

\section{Procesos y materialidad en el estudio del lenguaje en sociedad Processes and materiality in the study of language in society}

https://doi.org/10.1515/ijsl-2020-0067

Received August 26, 2020; accepted September 7, 2020

\begin{abstract}
In this brief essay, and making use of my own research in Peru, I raise two issues that I have been reflecting on throughout my career and that I believe constitute challenges in addressing language in society. The first refers to the importance of studying the processes of meaning production from an ethnographic perspective, and the second, to the centrality of articulating the production of these meanings with the material conditions of existence that make them possible or difficult. These two points are committed to combining ethnographic sociolinguistics and glotopolitics, as critical perspectives that are enhancing sociolinguistics in Latin America.
\end{abstract}

Keywords: critical sociolinguistics; ethnography; language ideologies; political economy

\section{Introducción}

Me formé como sociolingüista en la Universidad de Georgetown hace dos décadas bajo la asesoría de Ron Scollon y, desde ahí, siempre me he sentido en las fronteras disciplinarias y en los márgenes de la lingüística como disciplina. En este breve ensayo, quisiera señalar dos asuntos sobre los cuales he venido reflexionando a lo largo de mi carrera y que creo que constituyen desafíos para abordar el lenguaje en sociedad. El primero se refiere a la importancia de estudiar los procesos de producción de significados desde una perspectiva etnográfica; y el segundo, a la

Correction note: The English title "Processes and materiality in the study of language in society" was added after online publication March 11, 2021.

*Corresponding author: Virginia Zavala, Pontificia Universidad Católica del Perú, Lima, Peru, E-mail:vzavala@pucp.edu.pe 
centralidad de articular la producción de estos significados con las condiciones materiales de existencia que los posibilitan o dificultan. Para ilustrarlos, echaré mano de mis propias investigaciones, enmarcadas en una perspectiva crítica y etnográfica.

\section{Sobre los procesos}

El primer punto se conecta con una tensión existente entre estudiar ideologías como productos acabados que se revelan en textos o, más bien, como procesos que hay que abordar desde las prácticas sociales. Considero que esta tensión, que suelo observar entre mis estudiantes, se puede derivar de los desencuentros epistemológicos y metodológicos entre diferentes perspectivas del análisis del lenguaje en uso. Así por ejemplo, es bastante común analizar ideologías lingüísticas desde el paradigma de los estudios críticos del discurso (con mucha presencia en Latinoamérica), donde estas se tienden a asumir como creencias fijas que se analizan en textos, y no como procesos que se desarrollan en el marco de las acciones. Según Blommaert, el campo de la antropología lingüística y de los estudios críticos del discurso representan "two (or more) separate worlds - and a lot of untapped sources of mutual inspiration” (2005: 9). Creo que en Latinoamérica el desafío por lograr esta integración es aún mayor.

Esta necesidad de abordar los procesos, y no solo los productos, constituye una problemática que se viene discutiendo desde hace tiempo. Briggs (1992) ya mencionaba que las ideologías lingüísticas se suelen estudiar como un conjunto de creencias fijo, abstraído y circunscrito; que se distancia de las prácticas sociales y de la constitución de las ideologías en la acción. De otro lado, Woolard también ha llamado la atención sobre la importancia de abordar la construcción de los significados en curso: "Some linguistic anthropologists now worry that there is a trend toward the construal of ideology solely or primarily as metalinguistic discourse and a consequent lack of analytic attention to linguistic form and practice” (2008: 437). Si tomamos en cuenta que las ideologías remiten al ámbito de las ideas como también al de las prácticas y, por ende, de los procesos que constituyen el significado social de la actividad humana, nuestro objeto de estudio deberían ser, sobre todo, las prácticas y no solo los textos, tal como ya lo habían señalado los Scollon de forma insistente (2004). Esta centralidad de las prácticas ha llevado a priorizar categorías como las de "racialización” y "racializado" (sobre las de "raza” y "racismo"), pues ambas hacen alusión a los procesos semióticos e ideológicos a través de los cuales la raza llega a ser producida y cosificada a partir de las prácticas del lenguaje (Chun y Lo 2016). Creo que el abordaje de los procesos a partir de los cuales las formas lingüísticas se comienzan a ver como naturales a grupos racializados específicos puede 
complementar el importante desarrollo de los estudios del racismo en Latinoamérica desde las Ciencias Sociales (Zavala y Back 2017).

En mis propias investigaciones, he optado siempre por una perspectiva etnográfica para analizar los procesos indexicales por medio de los cuales las formas lingüísticas van articulándose con tipos de personas, situaciones y contextos en el marco de valoraciones específicas (Irvine y Gal 2000). Si bien el quechua se ha construido en el Perú como una lengua asociada a la pobreza, la ignorancia y la ruralidad, en la última década este vínculo indexical se ha venido reconfigurando a partir de nuevas dinámicas sociales, entre las que se encuentra un mayor poder adquisitivo de un gran sector de la población, un mayor acceso a la educación superior por parte de hijos de quechua hablantes y un discurso nacional asociado al fenómeno de la "Marca País". Este fenómeno busca reimaginar la nación dentro de un renovado sentido de unidad nacional y promover que los ciudadanos se conviertan en “embajadores” de su cultura en el marco de las expectativas de un proyecto económico neoliberal. Así por ejemplo, son los jóvenes hijos de quechua hablantes, ahora profesionales (o en vías de serlo), que viven en las ciudades, que están permanentemente conectados con las redes sociales, y que tienen una mayor consciencia y un discurso más elaborado sobre la opresión histórica de los hablantes de lenguas originarias, los que están liderando estos cambios en las representaciones (y prácticas) del quechua.

Este fenómeno revela varios niveles de discursos que se entrecruzan y que hay que analizar en toda su complejidad (Blommaert 2016). Si bien no ahondaré en esto por falta de espacio, sí compartiré un aspecto muy puntual que ha ido emergiendo en el trabajo etnográfico. Mientras observaba las clases de quechua que varios de estos jóvenes dictan en Lima, y también las interacciones que se desarrollan en línea en páginas conectadas con el uso de la lengua, pude darme cuenta que el quechua está ganando prestigio sólo en la medida en que se añade al conocimiento de un "buen" español y también del inglés, como si el "buen" español, pero sobre todo el inglés con aún más garantía, validaran el uso del quechua y le otorgaran un prestigio que hasta ahora no había tenido. Muchos jóvenes que están enseñando quechua en la ciudad suelen usar inglés en ciertos momentos de su clase, y muchas otras personas que interactúan online también usan recursos asociados al quechua y al inglés de forma simultánea. Esto muestra nuevas dinámicas identitarias en el Perú, en las que una subjetividad quechua urbana se está erigiendo desde nuevas indexicalidades. Es precisamente la perspectiva etnográfica la que provee un mayor poder explicativo para responder cómo, dónde, cuándo, con quién y, sobre todo, por qué se origina y circula una ideología lingüística. 


\section{Sobre las condiciones materiales}

Desde sus inicios, la sociolingüística fue un proyecto académico pero también político, interesado en mostrar el control desigual de los recursos simbólicos y materiales. Sin embargo, muchas veces tendemos a priorizar el análisis de las estructuras discursivas sin lograr conectar bien estos aspectos con la producción y reproducción del poder en relación al control de los recursos. La glotopolítica como perspectiva ha enfatizado la necesidad de priorizar lo anterior, en el marco de la comprensión de los procesos históricos. Sabemos que la materialidad del discurso es solo un correlato de condiciones materiales de existencia que posibilitan o dificultan su producción. Por eso, es fundamental estudiar esta materialidad discursiva en función de otros fenómenos más amplios y en diálogo con otras disciplinas.

Este punto se vincula, por ejemplo, con la despolitización del estudio de las ideologías lingüísticas, en el sentido de que a veces se las desvincula del ejercicio del poder en la sociedad. A pesar de que la perspectiva original de las ideologías lingüísticas siempre ha tomado muy en serio la noción de ideología y economía política, muchos trabajos utilizan la noción de ideologías lingüísticas como otra manera de hacer referencia a "idea sobre el lenguaje" de una forma bastante laxa. Se trata de una tensión que también suelo observar en mis estudiantes. Esto tiene como consecuencia que se diluya la fuerza del término y que todo pueda terminar constituyendo una ideología lingüística.

Este punto también ha sido ampliamente debatido desde el "giro" de la economía política en la sociolingüística crítica (Rosa y Burdick 2016) y la lingüística aplicada (Block 2017), a partir de los trabajos clásicos de Irvine y Gal. Sabemos que el lenguaje - siempre imbricado en procesos sociales- constituye un lugar central para la producción de diferencias sociales, y que estas no solo crean jerarquías sociales sino también el acceso diferenciado a recursos simbólicos y materiales. Esto supone ubicar el estudio de las manifestaciones discursivas en el contexto histórico, las lógicas del mercado, las ideologías patriarcales y el sistema postcolonial que las han producido (Duchêne 2020). Si volvemos nuevamente sobre las ideologías lingüísticas, podemos señalar que estas ideologías no se limitan a conceptos o creencias personales erróneos, sino que "(they are) always linked to the interests of differently positioned social actors and often embedded within institutionalized practices" (Lewis 2018: 4).

Aquí quisiera poner un ejemplo sobre algo que me pasó durante la cuarentena por la pandemia del Covid-19 en mayo del 2020. Fui invitada al programa “Aprendo en Casa”, transmitido por la televisión nacional peruana, para comentar 
el video “Los Castellanos del Perú” que realicé hace 16 años con unos colegas de mi universidad. La alusión a que el castellano estándar constituye una variedad lingüística construida a partir del poder de los grupos sociales privilegiados generó una reacción negativa de un sector conservador del país, que se opuso a que en la educación se hiciera referencia a los "grupos de poder” y a su responsabilidad en la reproducción de la desigualdad. Esto generó un escándalo mediático sin precedentes que terminó por provocar la renuncia de la Vice Ministra de Educación, el retiro del financiamiento al programa por parte de la empresa privada y la declaración del Ministro de Educación ante el Presidente de la República sobre el compromiso de su sector para seguir trabajando temas de discriminación y ciudadanía en la escuela. A partir de una avalancha de opiniones en los medios impresos y digitales (a veces contra mi persona), este debate reveló las disputas sobre el tipo de educación que necesitan los estudiantes, pero, sobre todo, sobre el sistema socioeconómico que debería regir en el país.

Este debate aparentemente sobre el lenguaje tuvo como agenda (no tan oculta) los intereses de un grupo económico neo conservador que desde hace décadas ejerce su poder sobre los gobiernos en el Perú y se asegura de que se mantenga una hegemonía neoliberal absoluta que sigue beneficiando a algunos y excluyendo a una gran mayoría. Como estrategia para mantenerse en el poder, y aludiendo implícitamente a los horrores ocurridos en las dos décadas de violencia política en el Perú (1980-2000), hoy se califica de "terroristas”, “comunistas” (o simplemente “terrucos") a cualquier persona que se atreva a cuestionar la hegemonía de lo dado. A partir del programa "Aprendo en Casa”, estos grupos vincularon una serie de signos para construir una diferenciación entre dos tipos de ciudadanos: aquellos que hablan sobre discriminación lingüística, traicionan a la patria y quieren hacerle daño a jóvenes estudiantes; y aquellos que creen que el castellano es solo uno, están a favor del "único" sistema que promueve crecimiento económico y hacen que el "Perú avance”. Esta diferenciación entre grupos sociales, y la permanente descalificación de cualquiera que aluda a la justicia social, tienen a su vez consecuencias materiales sobre los tipos de políticas que se priorizan en el país y las vidas de gran parte de la población.

Pienso que los dos puntos discutidos en este trabajo apuestan por combinar la sociolingüística etnográfica (Codó et al. 2012) y la glotopolítica (Del Valle 2017), como perspectivas que están potenciando la sociolingüística en Latinoamérica. Esto implica realizar análisis discursivos rigurosos, pero también abordar las condiciones históricas que permiten $\mathrm{u}$ obstaculizan las formaciones discursivas y acompañar in situ los procesos de construcción de los significados en las prácticas sociales. 


\section{References}

Block, David. 2017. Political economy in applied linguistics research. Language Teaching 50(1). 32-64.

Blommaert, Jan. 2005. Discourse: A critical introduction. Cambridge: Cambridge University Press.

Blommaert, Jan. 2016. From mobility to complexity in sociolinguistic theory and method. En Nikolas Coupland (ed.), Sociolinguistics. Theoretical debates, 242-259. Cambridge: Cambridge University Press.

Briggs, Charles. 1992. Linguistic ideologies and the naturalization of power in Warao discourse. Pragmatics 2. 387-404.

Chun, Elaine \& Adrienne Lo. 2016. Language and racialization. En Nancy Bonvillain (ed.), The Routledge handbook of linguistic anthropology, 220-233. Maiden, MA: Blackwell.

Codó, Eva, Adriana Patiño Santos \& Virginia Unamuno. 2012. Hacer sociolingüística etnográfica en un mundo cambiante: Retos y aportaciones de la perspectiva hispana. Spanish in Context 9(2). 167-190.

Del Valle, José. 2017. Glotopolítica y teoría del lenguaje. La perspectiva glotopolítica y la normatividad. Anuario de Glotopolítica 1.

Duchêne, Alexandre. 2020. Multilingualism: An insufficient answer to sociolinguistic inequalities. International Journal of the Sociology of Language 263. 91-97.

Irvine, Judith \& Susan Gal. 2000. Language ideology and linguistic differentiation. En Paul Kroskrity (ed.), Regimes of language: Ideologies, polities and identities, 35-84. Nuevo Mexico: School of American Research Press.

Lewis, Mark. 2018. A critique of the principle of error correction as a theory of social change. Language in Society 47(3). 325-346.

Rosa, Jonathan \& Christa Burdick. 2016. Language ideologies. En Ofelia García, Nelson Flores y Massimiliano Spotti (eds.), The Oxford handbook of language and society, 103-123. Oxford: Oxford University Press.

Scollon, Ron \& Suzanne W. Scollon. 2004. Nexus analysis. Discourse and the emerging internet. Nueva York: Routledge.

Woolard, Kathryn. 2008. Why dat now?: Linguistic-anthropological contributions to the explanation of sociolinguistic icons and change. Journal of SocioLinguistics 12(4). 432-452.

Zavala, Virginia y Michele Back. 2017. Racismo y lenguaje. Lima: Fondo Editorial de la Pontificia Universidad Católica del Perú. 